\title{
The role of intracavernous injection of vasoactive medications for the restoration of erection in spinal cord injured males: a three year follow up
}

\author{
DR Bodner MD, B Leffler RN, F Frost MD \\ Division of Urology and Department of Rehabilitation Medicine, Case Western Reserve \\ University School of Medicine, 2065 Albert Road, Cleveland, Ohio 44124, USA.
}

\begin{abstract}
Fifty-eight spinal cord injured men entered our self injection program for restoration of erections since the program began in September 1985. Early complications included sustained erection ( $>8$ hours) in 4 patients on 6 occasions requiring aspiration of the corpora cavernosa and injection of a dilute epinephrine solution $(1 / 100,000)$. Bruising at the site of injection was reported at least once by 10 patients. Blood draining from the urethra was noted in 3 patients when the urethra was inadvertently injected. Rigid erections were obtained in all but 5 patients that injected $(90 \%)$. Late complications included penile plaque in 3 patients $(15 \%)$. There were no abnormal liver function tests. Average time on the injection program was 2 years (range of 0.5 to 4.5 years). Thirty-one $(53 \%)$ of the patients have dropped out of the program, most doing so prior to the first injection or during the titration period. The average frequency of injection was twice a month. Injection therapy remains an effective and relatively safe alternative for selective patients with spinal cord injury.
\end{abstract}

Key words: impotence; spinal cord injury; intracavernous injections; papaverine.

\section{Introduction}

Obtaining and sustaining an erection sufficient for satisfactory intercourse is a frequent complaint of men with spinal cord injury. The ability to achieve reflex and psychogenic erections is related to the level of injury. ${ }^{1}$ Patients with upper motor neuron lesions obtain reflex erections but often have difficulty sustaining them to permit adequate intercourse. Patients with lower motor neuron lesions obtain either psychogenic erections, or no erection at all. Intracavernous injection of vasoactive drugs to produce erections has gained widespread use since 1982 when Virag reported that injection of papaverine, a smooth muscle relaxant, produced a fully rigid erection. ${ }^{2}$ Initial experience with intracorporal injection suggested that patients with neuropathic impotence achieve the best erections but are most susceptible to sustained erections. ${ }^{3-5}$ We report our longer term experience with 58 spinal cord injured men, followed up in our spinal cord injury unit, who entered the injection program.

\section{Materials and methods}

We admitted to the injection program 58 men, followed regularly through our spinal cord injury clinic, who were experiencing difficulty in obtaining or in maintaining an adequate erection. All patients had sustained a spinal cord injury at least one year prior to entering the program. Each patient had his medical history taken and a physical examination carried out by the urologist, and was interviewed by the spinal cord injury psychologist. Urinalysis, SMAC 20, and serum testosterone were obtained from all the patients who were considered to be good candidates for the injection program, and informed consent was obtained from each participant.

The age of the patient averaged 37.7 years (range 19-68 years). The interval between injury and inclusion in the program averaged 11.5 years (range $1-34$ years). There were 19 cervical, 32 thoracic, and 17 lumbar injuries with 44 of the lesions complete and 14 incomplete. Of the patients 31 were married or engaged, 25 were single and 2 
were divorced. Patients were seen on a weekly basis during the titration period and then every 2 months thereafter. Injections were begun with $7.5 \mathrm{mg}$ papaverine and gradually titrated to a dosage that produced an adequate erection. The patients were observed for 30 minutes after injection. Blood pressure was monitored and patients were instructed to return if the erection did not go down within 4 hours. Two adequate erections were required without producing a sustained erection ( $>4$ hours) before the patient was taught to perform self injection. Follow-up physical examinations were performed every 2 months and liver function tests were repeated every 4 months.

\section{Results}

Fifty-eight patients entered the program. Eight dropped out prior to receiving their first injection of papaverine. Rigid erections were obtained in 45 patients $(90 \%)$. Twenty-four of the patients received papaverine alone with a mean dosage of $15 \mathrm{mg}$ (range $5-30 \mathrm{mg}$ ). The combination of papaverine and phentolamine was administered to 26 of the patients with a mean papaverine dosage of $24 \mathrm{mg}$ (range $10-60 \mathrm{mg}$ ) and a mean dosage of phentolamine of $0.8 \mathrm{mg}$ (range $0.5-2 \mathrm{mg}$ ). Five patients achieved semi rigid erections that were insufficient for adequate intercourse. Further evaluation of these patients revealed 3 to have severe peripheral vascular disease, one venous leakage and one postpriapism fibrosis in a patient referred from an outlying institution after a sustained erection that required a shunt. The patients were followed up on the program from 0.5 to 4.5 years (mean 2 years). The average frequency of injection was twice a month (range 0-8). Early complications included a prolonged erection ( $>8$ hours) occurring in 4 patients (6 occasions) requiring aspiration of the corpora and injection of a dilute epinephrine solution $(1 / 100,000)$. Bruising was reported by 10 patients on at least one occasion. Three patients noted blood from the urethra when they inadvertently injected the urethra. Autonomic dysreflexia and also skin infection of the penis were not observed. Late complications included penile

plaque in 3 patients (15\%). One occurred after a patient sustained a penile fracture after falling on his erect penis. A second patient was noted to have fibrosis of the corpora cavernosa that did not respond to injection therapy after a shunt for priapism created by injection therapy at an outlying institution. No significant change was noted in the liver function tests on any of the patients.

\section{Drop out}

Thirty-one $(53 \%)$ of the patients dropped out within 4 years of beginning the injection program, with the greater number stopping prior to the first injection or during the drug titration period (Table 1). Reasons for drop out included inadequate erections in 5 patients, use of vasoconstrictive devices in 3 , injection complications in 2 , moving home in 7 , not having a current partner in 10 , and other medical problems. The patients that dropped out of the program injected themselves an average of twice a month, while those continuing injections averaged 3 times per month.

\section{Discussion}

Intracavernous injection of vasoactive medications has been performed only since 1982 . The long term complications are just being learned. Initial experience suggests that patients with neuropathic impotence respond best to injection of vasoactive medications. Yet it is the neuropathically impotent patient who is most prone to obtain a sustained erection with the potential for corporal fibrosis and refractory

Table 1 Drop out. Fifty-eight patients began program

Time of drop out

\section{Prior to first injection}

During drug titration

6 months

1 year

2 years

3 years

4 years

Total
No. of patients

$8(14 \%)$
$8(14 \%)$
$3(5 \%)$
$7(12 \%)$
$4(7 \%)$
$1(1 \%)$
0
$31(53 \%)$


impotence, as was seen in one of our patients injected with papaverine in an outlying institution and surgically shunted one week after onset of the erection. Erections in spinal cord injured patients must be reversed if they remain for more than 4 hours, to prevent this potential complication.

With careful observations, spinal cord injured patients can be expected to respond well with minimal morbidity to injection therapy. Yet it must be remembered that spinal cord injured patients are often young and may require many years of treatment for their erectile dsyfunction. We reported that plaques occurred in the penis of non neurogenically impotent men when the penis was injected a mean of 51 times at a mean frequency of 5.5 times per month. ${ }^{6}$ When the frequency of injection and dosage of medication was less, no plaque formed. ${ }^{6}$ In the non neurogenically impotent patients, we noted subtle plaque development in the penis in $53 \%$ of the patients. ${ }^{6}$ There was no true clinical relevance to the plaque; namely, the penis remained straight and the patient had no problem with intercourse. In our spinal cord injured patients, we noted development of penile plaque in only 3 patients $(15 \%)$. Two of the patients with penile plaque or scar developed this in a known manner: one after a postpriapism shunt and the other after fracturing his penis by falling in the erect penis, noting immediate detumescence and bruising but not presenting for follow up until one month after the injury. The latter patient subsequently noted a slight bend of the penis with erections. Perhaps the much lower incid- ence of plaque formation in the spinal cord injured population was because of the lower dosage of medication required for injection and the lower frequency of injection (average 2 per month in this series). However, longer follow up will be required to determine if the incidence of plaque formation increases and if the plaque has any clinical significance. No significant liver function abnormality was encountered in any patient. A high drop out rate of $53 \%$ from the injection program was noted with nearly one third of the patients dropping out prior to beginning home self injection. Many of the patients that dropped out stated that they were reassured just to know that injection therapy was available should they need it and although they had dropped out of the program by our protocol, many still considered themselves to be actively involved.

\section{Conclusion}

Spinal cord injured patients on intracorporal injection therapy achieved a high success rate with erections, were more prone to develop sustained erections, had a low frequency of use, a high drop out rate and a low incidence of long term complications. Injection therapy remains a relatively safe alternative when used carefully with close supervision for selective patients with spinal cord injury. Longer follow up is necessary to determine the significance of early plaque detection. Since spinal cord patients are often young and may require long term treatment, every effort should be made to minimize the morbidity of any of these newer treatment options.

\section{References}

1 Bors E, Comarr AE (1960) Neurological disturbances of sexual function with special reference to 529 patients with spinal cord injury. Urol Surv 10: 191.

2 Virag R (1982) Intracavernous injection of papaverine for erectile failure. Letter to the Editor. Lancet 2: 938.

3 Sidi AA, Cameron JS, Duffy LM and Lange PH (1986) Intracavernous drug-induced erections in the management of male erectile dysfunction: experience with 100 patients. J Urol 135: 704.

4 Wyndaele JJ, de Meyer JM, de Sy WA, Claessens H (1986) Intracavernous injection of vasoactive drugs, an alternative for treating impotence in spinal cord injury patients. Paraplegia 24: 271.

5 Bodner DR, Lindan R, Leffler E, Kursh ED, Resnick MI (1987) The application of intracavernous injection of vasoactive medications for erection in men with spinal cord injury. J Urol 138: 310-311.

6 Levine SB, Althof SE, Turner LA, Risen CB, Bodner DR, Kursh ED, Resnick MI (1989) Side effects of self-administration of intracavernous papaverine and phentolamine for the treatment of impotence. $J$ Urol 141: $54-57$. 